\title{
Respiratory Disorders in Acromegalic Patients
}

\author{
Valeria Mercuri', Tullia Villani' ${ }^{2}$, Denise Costa' ${ }^{1}$, Michela Mordenti², Tania D'Amico', \\ Paolo Palange $^{2}$, Patrizia Gargiulo ${ }^{1}$ \\ ${ }^{1}$ Department of Experimental Medicine, Endocrinology-Pituitary Disease, “Sapienza” University of Rome, Rome, Italy \\ ${ }^{2}$ Department of Public Health and Infectious Diseases, "Sapienza” University of Rome, Rome, Italy \\ Email: v.mercuri79@gmail.com, tulliavillani85@yahoo.it, denise.costa@libero.it, michelamordenti@tiscali.it, \\ damico.ta@gmail.com,paolo.palange@uniromal.it,patrizia.gargiulo@uniromal.it
}

How to cite this paper: Mercuri, V., Villani, T., Costa, D., Mordenti, M., D’Amico, T., Palange, P. and Gargiulo, P. (2019) Paper Title. International Journal of Clinical Medicine, 10, 553-564.

https://doi.org/10.4236/ijcm.2019.1010045

Received: September 16, 2019

Accepted: October 20, 2019

Published: October 23, 2019

Copyright $\odot 2019$ by author(s) and Scientific Research Publishing Inc. This work is licensed under the Creative Commons Attribution International License (CC BY 4.0).

http://creativecommons.org/licenses/by/4.0/ (c) (i) Open Access

\begin{abstract}
Purpose: To evaluate the prevalence and clinical performance of obstructive sleep apnoea syndrome (OSAS) in acromegalic patients, focusing on the possible correlation between alterations of pulmonary microcirculation and patient's clinical and hormonal parameters. Methods: We enrolled 22 acromegalic patients with apnea hypopnea index (AHI) $\geq 5$ if symptomatic, or $\geq 15$ in the absence of sleep related symptoms. Patients underwent the following evaluations: GH and IGF-1 serum levels, arterial blood gas test, spirometry, carbon monoxide diffusing capacity (DLCO), home-based cardiorespiratory sleep and pulmonary function test. Results: The prevalence of OSAS was $66.6 \%$. Patients with a severe form of OSAS appeared to be affected from acromegaly for more years than those with a lower severity of disease and those with acromegaly duration $>10$ years had significantly higher levels of glycated hemoglobin. We observed a positive correlation between GH level at diagnosis and DLCO value, showing that acromegalic patients have an increase in static and dynamic respiratory volumes and alveolar-capillary exchange surface compared to the general population. We also observed a positive correlation between baseline serum IGF-1 level and DLCO/VA and between years of disease and DLCO/VA, showing the association of increased lung volume and increase of the exchange surface. Conclusions: The results of this study add new information on lung volume and alveolar gas exchange in acromegaly. Our findings highlight the role of SSA as therapy with positive impact on complication and comorbidities of acromegaly.
\end{abstract}

\section{Keywords}

Acromegaly, Obstructive Sleep Apnoea Syndrome, Growth Hormone, Insulin-Like Growth Factor 1, Carbon Monoxide Diffusing Capacity 


\section{Introduction}

Acromegaly is a severe disease characterized by hypersecretion of growth hormone $(\mathrm{GH})$, which induces the synthesis of peripheral insulin-like growth factor 1 (IGF-1) after the closure of the epiphyseal bone. Acromegaly is usually the result of a GH-secreting pituitary adenoma, which results in anatomical changes and metabolic dysfunction. In rare cases, the disease is associated with excessive production of GH-releasing hormone (GHRH), familial syndromes, including multiple endocrine neoplasia type 1, McCune-Albright syndrome, familial acromegaly, and Carney's syndrome. Extrapituitary ectopic hypersecretion of GH has been reported in isolated cases of pancreatic islet-cell tumors or lymphoma. Clinical manifestations of acromegaly range from subtle signs of acral overgrowth, soft tissue swelling, arthralgias, jaw prognathism, fasting hyperglycemia, and hyperhidrosis to florid osteo-arthritis, frontal bone bossing, diabetes mellitus, hypertension, respiratory and heart failure [1] [2].

In recent years, there has been mounting interest in respiratory disorders during sleep in patients with acromegaly. In fact the anatomical changes commonly occurring in patients with acromegaly, such as facial soft-tissue thickening (especially of the palate and uvula), may induce the onset of the obstructive sleep apnoea syndrome (OSAS), due to hypercollapsibility of the posterior and lateral hypopharyngeal walls, as well as to hypertrophy of the tongue [3].

Nocturnal breathing disorders affect the majority of patients with acromegaly. Sleep apnoea syndrome (SAS), diagnosed by polysomnography, has been reported in about $70 \%$ of patients with active acromegaly disease; the frequency, however, ranges from $45 \%$ to $80 \%$ [3] [4]. SAS can be central or mixed, but it is obstructive in most cases.

OSAS is a highly prevalent sleep disorder characterized by a transient cessation in breathing (apnea) or a significant reduction in breathing amplitude (hypopnea), due to recurrent episodes of upper airway obstruction and subsequent recurrent arousal during sleep. This syndrome is characterized by daytime sleepiness, non-restorative sleep, fatigue, insomnia, loud snoring, witnessed breathing interruptions, morning headaches, and recurrent awakenings due to gasping or choking in the presence of at least five obstructive respiratory events (apneas, hypopneas, or respiratory effort-related arousals) per hour of sleep. The presence of 15 or more obstructive respiratory events per hour of sleep in absence of sleep-related symptoms is also sufficient for a diagnosis of OSAS, due to the greater association of this severe degree of obstruction with important complications, such as increased cardiovascular and metabolic disease risk [5]. Early markers of pulmonary damage may however have a great importance in the diagnosis of OSAS, in order to institute prompt treatment.

The principal aim of this study was to further evaluate the prevalence and secondly the clinical performance of OSAS in relation to duration of acromegalic disease. Moreover, we assessed the possible correlation between the alterations in gaseous exchanges at the level of pulmonary microcirculation and the clinical 
and hormonal parameters, to identify possible early markers of pulmonary performance damage.

\section{Patients and Methods}

\subsection{Patients}

Population: Patients referring to the Metabolic Emergencies-Pituitary Disease Unit of the Department of Experimental Medicine, Policlinico Umberto I- "Sapienza" University of Rome, were evaluated in this study. The local Ethical Committee approved the study design, and all patients signed an informed consent to the use of their data for research purposes.

Inclusion criteria: patients with the diagnosis of acromegaly (in agreement to consensus on the medical treatment of acromegaly) (November 2014) [6].

Exclusion criteria: Patients affected from major systemic diseases such as malignant cancer, immune disorders, acute cardiovascular and cerebrovascular diseases or other disorders correlated with apnea (e.g. hypothyroidism), or those with intake of psychotropic drugs, alcoholism, smoking and other abuses, were excluded. All patients were biochemically targeted for any other hormone deficiency.

Baseline characteristics of study population are described in Table 1.

\subsection{Diagnosis of Acromegaly}

The diagnosis of acromegaly was performed on the bases of the latest available guidelines, which, in patients with elevated or equivocal serum IGF-1 concentrations, recommend confirmation of the diagnosis by finding lack of suppression of $\mathrm{GH}$ to $<0.4 \mu \mathrm{g} / \mathrm{l}$, following documented hyperglycemia during an oral glucose load (2 hours after $75 \mathrm{~g}$ of oral glucose) [6] [7] [8].

Table 1. Clinical data.

\begin{tabular}{cc}
\hline \multicolumn{2}{c}{ Study population } \\
\hline Number of patients (11 M and 11 F) \\
Age (years) & 22 \\
Mean DD & $12.9 \pm 8.9 \pm 19.5$ \\
& $<10$ years \\
& $>10$ years in $12 / 22$ patients \\
Incidence of OSAS & $66.6 \%(15 / 22)$ \\
Mild grade & $40 \%(6 / 15)$ \\
Moderate grade & $26.6 \%(4 / 15)$ \\
Severe grade & $33.3 \%(5 / 15)$ \\
HbAlc (\%) in OSAS patients with DD $>10$ years & $6.2 \pm 1.4$ \\
HbAlc (\%) in OSAS patients with DD $<10$ years & $5.1 \pm 0.5$
\end{tabular}

DD: Duration of disease from diagnosis; HbAlc: glycated hemoglobin; OSAS: Obstructive Sleep Apnoea Syndrome. 
Biochemical control of disease was defined on the basis of the determination of IGF-1, age-related and expressed in $\mathrm{ng} / \mathrm{ml}[6]$. GH concentrations were not used for the evaluation of the biochemical control of disease since seven patients were treated with pegvisomant [6].

\subsection{IGF-1 and GH Serum Assay}

The determination of GH and IGF-1 run with chemiluminescent immunometric assay (SIEMENS Immunolite 2000) was performed at the Laboratory of Clinical Pathology of the Policlinico Umberto I.

The patients were referred to our Sleep Disorders Unit and underwent blood sampling (for the assessment of GH and IGF-1 serum levels), arterial blood gas test (ABG), spirometry, carbon monoxide diffusing capacity (DLCO) and home-based cardiorespiratory sleep. The study protocol has been defined in agreement with the ethical principles of the Helsinki Declaration. All patients gave written informed consent for their participation in the study.

\subsection{Home-Based Cardiorespiratory Sleep Study}

A home-based cardiorespiratory sleep study was performed by using a SOMNO screen device (SOMNO medics-GmbH, Randersacker, Germany). The study recorded the following parameters: chest and abdominal movements, nasal flow, body position, oxygen saturation, snoring, and heart rate. Daytime sleepiness was assessed using the Epworth Sleepiness Scale (ESS) [9], and symptoms such as daytime sleepiness, non-restorative sleep, fatigue, insomnia, snoring, witnessed breathing interruptions, morning headaches, and recurrent awakenings due to gasping or choking were recorded. Cardiorespiratory analysis was examined according to the American Academy of Sleep Medicine (AASM) 2007 guidelines [10].

Apnea was defined as the cessation of airflow for a period $\geq 10 \mathrm{~s}$, and hypopnea was defined either as the reduction of at least $30 \%$ of the airflow associated with oxygen desaturation $\geq 4 \%$ or as a $\geq 50 \%$ airflow reduction with a $\geq 3 \%$ oxygen desaturation for $\geq 10 \mathrm{~s}$. From each recording, we assessed the Apnea Hypopnea Index (AHI; number of apneas and hypopneas per hour), percentage of total sleep time passed with an oxygen saturation $<90 \%(\mathrm{~T}<90 \%)$ and the Oxygen Desaturation Index (ODI; number of oxygen desaturations per hour), as parameters of disease severity. Inclusion criteria were AHI $\geq 5$ in symptomatic patients or $\mathrm{AHI} \geq 15$ in the absence of sleep related symptoms.

\subsection{Pulmonary Function Test}

Pulmonary function data were performed by standardized techniques using an automated pulmonary function testing system (COSMED PFT, Pavona Italy). The following tests were collected and standardized as percentages of predicted normal values: spirometry, nitrogen washout and single-breath diffusing capacity (DLCO) [10] [11]. 


\section{Statistical Analysis}

All data were tested to verify if assumptions of normality were true. The assumptions of normality could not be assumed within the data set particularly due to the small sample size. Therefore, nonparametric tests were used. Continuous data were compared using Wilcoxon rank-sum test. The Mann-Whitney $\mathrm{U}$ test or the Kruskal-Wallis was used to assess differences between the two groups (target and no target patients) and three groups according to OSAS severity (mild, moderate and severe). Spearman's rank order correlation coefficient ( $r$ ) was used to test associations between numerical variables. Data are reported as mean \pm standard error $(\mathrm{SE})$. P values $<0.05$ were considered significant. Multivariate analysis was applied for the estimation of the relationships between polysomnography variables, spirometry data and GH and IGF-1 serum levels. A commercial software (SPSS version 20.0) was used for statistical analysis.

\section{Results}

We selected 22 acromegalic patients, out of a total of 180 attending our Center, on the basis of symptoms or signs of respiratory disorders, evaluating among these the presence or absence of OSAS and the degree of severity.

The study included 22 acromegalic patients (11 males and 11 females). Table 1 shows baseline characteristics of study population.

At enrolment, the mean duration of disease from diagnosis (DD) was $12.9 \pm$ 8.9 years (>10 years in 12/22 subjects). All patients presented biochemical control of acromegalic disease, with normal age-adjusted levels of IGF-1.

The prevalence of OSAS was $66.6 \%$ (15/22 of cases).

In more details, $40 \%$ of them (6/15) showed a mild OSAS grade (AHI $10.6 \pm 2$ events per hour), 26.6\% (4/15) a moderate grade (AHI $19.9 \pm 4.4$ events per hour) and 33.3\% (5/15) a severe OSAS (AHI $41.1 \pm 9.9$ events per hour) (Table 1).

When stratifying patients according to OSAS severity (mild, moderate, severe), we observed that severe OSAS patients appear to have been affected by acromegaly for several years (determined illness plus suspected disease) $(20.4 \pm$ 9.4 years vs $14.7 \pm 5.2$ vs $34.1 \pm 17.5 ; \mathrm{p}=0.05)$.

Significantly higher values of glycated hemoglobin (HbA1c) were found in OSAS patients with acromegaly duration $>10$ years compared with those with $<10$ years of disease $(6.2 \pm 1.4$ vs $5.1 \pm 0.5 ; \mathrm{p}=0.01)($ Table 1$)$

Spirometry data of our patients showed the following values: forced vital capacity (FVC) (L) $4.32 \pm 1.6$, FVC $\% 116.3 \pm 21.6$, forced expiratory volume in 1 second (FEV1) (L) $3.3 \pm 1.7$, FEV1 \% $110.4 \pm 21$, FEV1/FVC $\% 77.67 \pm 7.7$, total lung capacity (TLC) \% 105.2 \pm 27.9 , DLCO \% $100 \pm 27.9$.

We disclosed a positive correlation between serum $\mathrm{GH}$ level at the time of the acromegaly diagnosis and DLCO value $(110.1 \pm 27.9)(p=0.04)$. A further positive correlation was demonstrated between baseline serum IGF-1 level and 
DLCO/alveolar volume (VA) value and between years disease (illness plus suspected disease) and DLCO/VA $(103.5 \pm 17.9 ; \mathrm{p}=0.04)$.

A negative correlation between the average of the last three determinations of serum IGF-1 levels, the values of serum bicarbonates $\left(\mathrm{HCO}^{3-}\right)(26.5 \pm 2.1 ; \mathrm{p}=$ $0.04)$ and carbon dioxide $\left(\mathrm{pCO}_{2}\right)(40.6 \pm 4.3 ; \mathrm{p}=0.019)$ was reported in all patients included in the study.

Lastly, in target patients the average of the last three determinations of serum IGF-1 level correlated negatively with the partial pressure of $\mathrm{pCO}_{2}$, determined at the time of enrolment with arterial blood gas test $(41 \pm 4.1 \mathrm{mmHg} ; \mathrm{p}=0.01)$.

\section{Discussion}

OSAS is a common disorder characterized by recurrent episodes of apnea or hypopnea, due to total or partial pharyngeal collapse and temporary upper airway obstruction during sleep, resulting in frequent episodes of hypoxemia and hypercapnia. Some endocrine and metabolic disorders (obesity, acromegaly, hypothyroidism, etc.) are associated with a high frequency of OSAS, and treatment of the underlying endocrine disorder can improve sleep disorders [12] [13] [14] [15].

Two hypothesis was proposed to explain the high prevalence of OSAS in acromegaly: 1) the influence of abnormal levels of pituitary hormones on impaired respiratory control; 2) the anatomical changes in the upper airways body changes are derived especially from skeletal growth and soft tissue enlargement, which is subtle in the early stage of disease [16] [17].

Facial changes include anatomical disorders (large lips and noses, macroglossia, mandibular overgrowth with prognathism, maxillary widening ...). Dostalova S. et al. argue that these anatomical disorders are not sufficient to cause OSAS, while soft tissue thickening is the more evident contributor to OSAS onset [18].

In several prospective or retrospective studies, the prevalence of OSAS in acromegaly is around $69 \%$ [19], vs $66.6 \%$ in ours. In more details, $40 \%$ affected by mild OSAS, $26.6 \%$ by moderate and $33.3 \%$ by severe. Roemmler J. et al. showed a positive correlation between respiratory disturbance index (RDI) and acromegalic disease activity, but not with the duration of the disease [20]. Another study reported a positive correlation between the severity of sleep-disordered breathing and duration of acromegaly in patients with active disease. Stratifying OSAS patients according to disease severity we observed that severe OSAS patients appear to be affected from acromegaly for more years than those with a lower severity of disease [21] [22].

Previous studies suggest that enhanced lung growth in acromegalic patients is associated with either a normal or above normal pulmonary transfer factor in pulmonary function test. Brody et al. suggest either alveolar hypertrophy or hyperplasia as the mechanism for lung growth in this condition. In fact, normal postnatal lung growth occurs in two major stages: the former, primarily asso- 
ciated with an increase in alveolar numbers, the latter correlated with an increase of alveolar size. Growth hormone excess resulted in the formation of new alveoli and new surface for gas exchange, therefore diffusing capacity (DL) should be increased in the acromegalic subjects. On the other hand, normal DL suggests that lung growth was associated with increased alveolar size; the increased alveolar volume or size of the pulmonary capillary bed was offset by the effects of increased cell size and interstitial tissue in impeding diffusion [23].

In our study we found a positive correlation between serum GH level at acromegaly diagnosis and DLCO. Our results showed that the static and dynamic respiratory volumes and alveolar-capillary exchange surface are increased if compared with the general population (adjusted for gender, age and weight) as shown by TLC\%, FEV1 (in liters and \%), FVC (in liters and \%) and DLCO\%.

Another hormone involved in tissue growth is IGF-1, which is generally accepted as a central mediator of metabolic, endocrine, and anabolic effects of GH. Moreover, an association of circulating IGF-I levels and effects on muscular strength of rehabilitation programs in patients with chronic obstructive pulmonary disease has been described [24] [25].

Glaser et al. reported a positive association between IGF-1 serum levels and higher lung volumes in men of all ages and women older than 50 years. IGF-1 serum values or increasing lung volumes were not associated with increased strength of respiratory muscles [26].

To this end, our results show positive correlation between baseline serum IGF-1 levels, DLCO/VA value and between years of disease (illness plus suspected disease) and DLCO/VA showing the association of increased lung volume and increase of the exchange surface (alveolar hypertrophy or hyperplasia).

The duration of the disease, as expected, plays an important role on the alterations of respiratory function in acromegalic patient. In fact, our data show that longer duration of illness is correlated with increased incidence of OSAS.

IGF-1 exerts multiple physiological effects on the vascular system, including proliferative, hypertrophic, survival, vasomotor and metabolic effects. The expression of IGF-1, IGF-1R, and IGFBPs in the blood vessels is regulated by multiple factors, including growth factors, cytokines, lipoproteins, reactive oxygen species and hemodynamic forces. The close interaction between IGF-1 and other growth factors system at the level of receptor-ligand and at the level of post-receptor signaling pathways has important implications in the understanding of the involvement of IGF-1 system in vascular diseases. Although recent studies have indicated that IGF-1 has a strong effect on angiogenesis [27] [28], it has been reported that IGF-1 can induce angiogenesis in skeletal muscle and brain tissue. This mechanism may also be present in the lung, thus justifying the increase in DLCO in patients with OSAS by increased alveolar capillarization.

Therefore, some authors demonstrated in male volunteers with high IGF-1 levels ratio values higher FEV1 and FVC values [26].

Increase in lung size has been described in acromegalic patients, but data on respiratory muscle function and control of breathing are relatively scant. Some 
patients with acromegaly show decreased respiratory muscle strength, although the magnitude of this change has not been significantly correlated with the increase in lung volume, or with measurements of arterial $\mathrm{PaCO}_{2}$ and $\mathrm{PaO}_{2}$ ) [29]. Peripheral (muscular) factors appear to modulate a normal central motor output to give a more rapid pattern of breathing. Varying levels of hypoxemia with increased alveolar-arterial oxygen gradients have been described in patients with acromegaly, thereby suggesting disturbance of the ventilation/perfusion relationship [30].

As a further support of a possible alteration of the ventilation/perfusion and of a more rapid pattern of breathing, our data showed a negative correlation between the average of the last three determinations of serum IGF-1 level and the concentration of serum bicarbonates $\left(\mathrm{HCO}^{3-}\right)$ and $\mathrm{CO}$. Last, in target patients the average of the last three determinations of serum IGF-1 level correlates negatively with the partial pressure of carbon dioxide, determined at enrolment with arterial blood gas test. Given the trend of bicarbonate and arterial carbon dioxide values in relation to hormonal IGF-1 values, we could speculate that in acromegalic patients the anatomical and functional changes of the lung can lead to a pattern of hyperventilation, despite the present diagnosis of OSAS. Different studies report the effects of GH/IGF-1 administration on the renal and systemic regulation of acid-base homeostasis.

The finding that $\mathrm{GH}$ administration results in markedly increased rates of renal hydrogen secretion and sodium chloride reabsorption, sufficient to cause these effects: 1) an increase in net acid excretion despite reclamation of an increased filtered load of bicarbonate; 2) an expanded extracellular fluid volume, as evidenced by weight gain and sodium retention despite an increased filtered sodium load, cannot be explained by the effects of enhanced mineral or glucocorticoid activities which decreased in response to GH [31] [32].

In contrast to these data we found in the acromegalic group bicarbonate values not very high compared with hormonal values, likely because such data were biased by the use of specific drugs such as somatostatin analogues, bromocriptine and receptor antagonists for the hormone growth or because the patients had been subjected to surgical intervention.

\section{Conclusions}

Our data are consistent with current literature on the prevalence, disease duration, body mass index (BMI), gender, neck circumference and Epworth Score of OSAS in patients with acromegaly, and do not show a statistically significant correlation with disease control. They also add new information on lung volumes and the alveolar gas exchange.

In relation to the therapy in acromegalic patients with OSAS, there is an unambiguous suggestion. Our study, however, has highlighted the role of the somatostatin analogues (SSAs) as therapy with a positive impact on complications and comorbidities of acromegalic disease. In the respiratory system, in fact, these molecules reduce the tissue turgidity and the sensitivity of chemoreceptors to 
hypoxia by improving breathing. Moreover, the effectiveness of SSAs as antinociceptive and analgesic on headache symptoms is underlined, further to the already known effects of shrinkage of pituitary adenoma and biochemical control of disease. For this reason, we are evaluating for a subsequent work the comparison of respiratory parameters in naively acromegalic patients versus patients in therapy with SSAs.

As recommended since the guidelines of Versailles in 2003 [7], given the prevalence of OSAS in acromegaly and associated comorbidities, it is mandatory to perform a polysomnographic examination in all patients with acromegaly at diagnosis and during the follow-up. In fact, since $25 \%$ of the mortality is due to respiratory disorders, early diagnosis of OSAS not only improves life expectancy and quality of life of patients, but also contributes to the reduction of cardiovascular risk with considerable impact on health care costs.

\section{Acknowledgements}

Editorial assistance for the preparation of this manuscript was provided by Luca Giacomelli, PhD Ambra Corti, and Pascal Vignally, M.D. on behalf of Content Ed Net; this assistance was supported by Novartis.

\section{Funding}

No funding was received for the preparation of this manuscript.

\section{Ethical Approval}

All procedures performed in studies involving human participants were in accordance with the ethical standards of the institutional and/or national research committee and with the 1964 Helsinki declaration and its later amendments or comparable ethical standards.

\section{Informed Consent}

Informed consent was obtained from all individual participants included in the study.

\section{Conflicts of Interest}

All the authors declare they have no conflict of interest.

\section{References}

[1] Melmed, S. (2006) Acromegaly. The New England Journal of Medicine, 355, 2558-2573. https://doi.org/10.1056/NEJMra062453

[2] Davì, M.V. and Giustina, A. (2012) Sleep Apnea in Acromegaly: A Review on Prevalence, Pathogenetic Aspects and Treatment. Expert Review of Endocrinology and Metabolism, 7, 55-62. https://doi.org/10.1586/eem.11.82

[3] Vannucci, L., Luciani, P., Gagliardi, E., Paiano, S., Duranti, R., et al. (2013) Assessment of Sleep Apnea Syndrome in Treated Acromegalic Patients and Correlation of Its Severity with Clinical and Laboratory Parameters. Journal of Endocrinological 
Investigation, 36, 237-242.

[4] Fatti, L.M., Scacchi, M., Pincelli, A.I., Lavezzi, E. and Cavagnini, F. (2001) Prevalence and Pathogenesis of Sleep Apnea and Lung Disease in Acromegaly. Pituitary, 4, 259-262. https://doi.org/10.1023/A:1020702631793

[5] Berry, R.B., Budhiraja, R., Gottlieb, D.J., Gozal, D., Iber, C., et al. (2012) Rules for Scoring Respiratory Events in Sleep: Update of the 2007 AASM Manual for the Scoring of Sleep and Associated Events. Deliberations of the Sleep Apnea Definitions Task Force of the American Academy of Sleep Medicine. Journal of Clinical Sleep Medicine, 8, 597-619. https://doi.org/10.5664/jcsm.2172

[6] Katznelson, L., Laws, E.R., Melmed, S., Molitch, M.E., Murad, M.H., et al. (2014) Acromegaly: An Endocrine Society Clinical Practice Guideline. The Journal of Clinical Endocrinology \& Metabolism, 99, 3933-3951.

https://doi.org/10.1210/jc.2014-2700

[7] Giustina, A., Casanueva, F.F., Cavagnini, F., Chanson, P., Clemmons, D., et al. (2003) Diagnosis and Treatment of Acromegaly Complications. Journal of Endocrinological Investigation, 26, 1242-1247. https://doi.org/10.1007/BF03349164

[8] Melmed, S., Casanueva, F.F., Klibanski, A., Bronstein, M.D., Chanson, P., et al. (2012) A Consensus on the Diagnosis and Treatment of Acromegaly Complications. Pituitary, 16, 294-302. https://doi.org/10.1007/s11102-012-0420-X

[9] Johns, M.W. (1991) A New Method for Measuring Daytime Sleepiness: The Epworth Sleepiness Scale. Sleep, 14, 540-545. https://doi.org/10.1093/sleep/14.6.540

[10] Epstein, L.J., Kristo, D., Strollo, P.J., Friedman, N., Malhotra, A., et al. (2009) Clinical Guideline for the Evaluation, Management and Long-Term Care of Obstructive Sleep Apnea in Adults. Journal of Clinical Sleep Medicine, 5, 263-276.

[11] Commissione Paritetica Associazione Italiana Medicina del Sonno (AIMS), Associazione Italiana Pneumologi Ospedalieri (AIPO) (2001) Linee guida di procedura diagnostica nella sindrome delle apnee ostruttive nel sonno dell'adulto.

http://www.sonnomed.it/linee guida.htm

[12] Attal, P. and Chanson, P. (2010) Endocrine Aspects of Obstructive Sleep Apnea. The Journal of Clinical Endocrinology \& Metabolism, 95, E483-E495. https://doi.org/10.1210/jc.2009-1912

[13] Guo, X., Gao, L., Zhao, Y., Wang, M., Jiang, B., et al. (2018) Characteristics of the Upper Respiratory Tract with Acromegaly and Correlations with Obstructive Sleep Apnoea/Hypopnea Syndrome. Sleep Medicine, 48, 27-34. https://doi.org/10.1016/j.sleep.2018.04.011

[14] De Menis, E., Giustina, A., Colao, A., Degli Uberti, E., Ghigo, E., et al. (2011) Assessment of the Awareness and Management of Sleep Apnea Syndrome in Acromegaly. The COM.E.TA (Comorbidities Evaluation and Treatment in Acromegaly) Italian Study Group. Journal of Endocrinological Investigation, 34, 60-64. https://doi.org/10.1007/BF03346696

[15] Ceccato, F., Bernkopf, E. and Scaroni, C. (2015) Sleep Apnea Syndrome in Endocrine Clinics. Journal of Endocrinological Investigation, 38, 827-834. https://doi.org/10.1007/s40618-015-0338-Z

[16] Rosenow, F., McCarthy, V. and Caruso, A.C. (1998) Sleep Apnoea in Endocrine Diseases. Journal of Sleep Research, 7, 3-11. https://doi.org/10.1046/j.1365-2869.1998.00086.x

[17] Cadieux, R.J., Kales, A., Santen, R.J., Bixler, E.O. and Gordon, R. (1982) Endoscopic Findings in Sleep Apnoea Associated with Acromegaly. The Journal of Clinical Endocrinology \& Metabolism, 55, 18-22. https://doi.org/10.1210/jcem-55-1-18 
[18] Dostalova, S., Sonka, K., Smahel, Z., Weiss, V., Marek, J., et al. (2001) Craniofacial Abnormalities and Their Relevance for Sleep Apnoea Syndrome Aetiopathogenesis in Acromegaly. European Journal of Endocrinology, 144, 491-497. https://doi.org/10.1530/eje.0.1440491

[19] Davi, M.V., Dalle Carbonare, L., Giustina, A., Ferrari, M., Frigo, A., et al. (2008) Sleep Apnoea Syndrome Is Highly Prevalent in Acromegaly and Only Partially Reversible after Biochemical Control of the Disease. European Journal of Endocrinology, 159, 533-540. https://doi.org/10.1530/EJE-08-0442

[20] Roemmler, J., Gutt, B., Fischer, R., Vay, S., Wiesmeth, A., et al. (2012) Elevated Incidence of Sleep Apnoea in Acromegaly-Correlation to Disease Activity. Sleep and Breathing, 16, 1247-1253. https://doi.org/10.1007/s11325-011-0641-7

[21] Weiss, V., Sonka, K., Pretl, M., Dosta’lova', S., Klozar, J., et al. (2000) Prevalence of the Sleep Apnea Syndrome in Acromegaly Population. Journal of Endocrinological Investigation, 23, 515-519. https://doi.org/10.1007/BF03343767

[22] Tsoi, U.A., Sviryaev, Y.V., Korostovtseva, L.S., Semenov, A.P., Vaulina, D.A., et al. (2015) Clinical Features of Obstructive Sleep Apnea Syndrome in Patients with Acromegaly. Terapevticheskii Arkhiv, 87, 47-52. https://doi.org/10.17116/terarkh201587447-52

[23] Brody, J.S., Fisher, A.B., Gocmen, A. and DuBois, A.B. (190) Acromegalic Pneumonomegaly: Lung Growth in the Adult. Journal of Clinical Investigation, 49, 1051-1060. https://doi.org/10.1172/JCI106321

[24] Creutzberg, E.C. and Casaburi, R. (2003) Endocrinological Disturbances in Chronic Obstructive Pulmonary Disease. European Respiratory Journal, 46, 76s-80s. https://doi.org/10.1183/09031936.03.00004610

[25] Vogiatzis, I., Stratakos, G., Simoes, D.C., Terzis, G., Georgiadou, O., et al. (2007) Effects of Rehabilitative Exercise on Peripheral Muscle TNF, IL-6, IGF-I and MyoD Expression in Patients with COPD. Thorax, 62, 950-956. https://doi.org/10.1136/thx.2006.069310

[26] Glaser, J., Friedrich, N., Ewert, R., Schäper, C., Nauck, M., et al. (2009) Association between Serum Insulin-Like Growth Factor (IGF) I and IGF Binding Protein-3 and Lung Function. The Journal of Clinical Endocrinology \& Metabolism, 94, 2452-2458. https://doi.org/10.1210/jc.2008-2662

[27] Dobrucki, L.W., Tsutsumi, Y., Kalinowski, L., Dean, J., Gavin, M., et al. (2010) Analysis of Angiogenesis Induced by Local IGF-1 Expression after Myocardial Infarction Using MicroSPECT-CT Imaging. Journal of Molecular and Cellular Cardiology, 48, 1071-1079. https://doi.org/10.1016/j.yjmcc.2009.10.008

[28] Rabinovsky, E.D. and Draghia-Akli, R. (2004) Insulin-Like Growth Factor I Plasmid Therapy Promotes in Vivo Angiogenesis. Molecular Therapy, 9, 46-55. https://doi.org/10.1016/j.ymthe.2003.10.003

[29] Iandelli, I., Gorini, M., Duranti, R., Bassi, F., Misuri, G., et al. (1997) Respiratory Muscle Function and Control of Breathing in Patients with Acromegaly. European Respiratory Journal, 10, 977-982. https://doi.org/10.1183/09031936.97.10050977

[30] Luboshitzky, R. and Barzilai, D. (1980) Hypoxemia and Pulmonary Function in Acromegaly. The American Review of Respiratory Disease, 121, 471-475. https://doi.org/10.1164/arrd.1980.121.3.471

[31] Kamenicky, P., Viengchareun, S., Blanchard, A., Meduri, G., Zizzari, P., et al. (2008) Epithelial Sodium Channel Is a Key Mediator of Growth Hormone-Induced Sodium Retention in Acromegaly. Endocrinology, 149, 3294-3305. 
https://doi.org/10.1210/en.2008-0143

[32] Sicuro, A., Mahlbacher, K., Hulter, H.N. and Krapf, R. (1998) Effect of Growth Hormone on Renal and Systemic Acid-Base Homeostasis in Humans. American Journal of Physiology, 274, F650-F657.

https://doi.org/10.1152/ajprenal.1998.274.4.F650 